\title{
Using Ground Penetrating Radar (GPR) to Evaluate Air Voids Content in Hot Mix Asphalt
}

Pejman Dehdezi

p.dehdezi@fugro.com

Fugro, Doha, Qatar

\begin{abstract}
Air voids content is one of the most important characteristics of Hot Mix Asphalt (HMA). Air voids content that is either too high or too low can negatively impact pavement life and lead to deteriorations such as fatigue cracking, rutting, raveling, bleeding, and moisture damage. Currently destructive testing, such as coring, is typically conducted to evaluate in-situ air voids content of placed HMA. Asphalt coring, by its nature, is highly localized and therefore can easily miss localize areas of low quality. In addition, in large projects, there are some concerns expressed on the aesthetic deficiencies with respect to the number of cores in the wearing course and the introduction of potential weak spots within the pavement structure. In other methods of air voids determination, such as nuclear density gauge, there are also concerns regarding safety, costs associated with radioactive material licensing and requirements for closing off work areas. In this paper the use of a "GSSI PaveScan RDM" GPR model for non-destructive assessment of insitu air voids in HMA is investigated. In-situ air voids content can be determined through plotting of dielectric changes with measured cores' air voids content. This technique provides an opportunity for continuous measurement of air voids in HMA and, at the same time, faster and reliable in-situ data for QA/QC. These benefits can significantly accelerate pavement construction and so this paper looks to proliferate the adoption of such practice. The case study on Al Khor Expressway, one of the major expressways in Qatar, has demonstrated good repeatability and correlation with measured air voids content.
\end{abstract}

Keywords: Ground Penetrating Radar (GPR); Hot Mix Asphalt (HMA); Air voids; Quality assurance; Non-destructive testing

\section{INTRODUCTION}

The air voids content of HMA, achieved by contractors at the time of construction, is the most important factor that affects the long-term durability of the pavement. The compaction process on site is the practice to control the air voids content of the HMA. In Qatar, properly designed HMA should have an air voids content in the range of $5 \%$ to $8 \%$ (QCS, 2014).

The primary performance concern of an HMA with low in-place air voids content could be deformation resistance (or rutting). Previous research shows that significant rutting was likely to occur once the in-place air voids fall below 3\% (Brown, 1990). On the other hand, the primary performance concern of high in-place air voids content would be reduction in pavement fatigue life. Several researchers have reported that fatigue properties can be reduced by 30 to $40 \%$ for each $1 \%$ increase in air voids content from 
5 to $8 \%$ (Linden et.al., 1989). Accelerated ageing, moisture damage, raveling, bleeding, decrease in stiffness and strength are other pavement distresses that would occur as a result of HMA air voids content that is either too high or too low.

Currently destructive methods are the most common methods for determination of density and air voids content in HMA. Many road owners specify destructively cutting cores in the pavement to determine air voids content of HMA. The drawbacks of destructive pavement coring are that the locations are randomly chosen, the process is destructive and relatively expensive, and it is labor intensive. Most importantly, it is time consuming and does not provide immediate feedback to the contractor on the HMA air voids content. Furthermore, aesthetic deficiencies with respect to the number of cores in the wearing course and the introduction of potential weak spots within the pavement structure are of concern.

Other existing technologies such as nuclear density gauges and electromagnetic density indicators (Figure 1) are alternative techniques to pavement coring that are currently used in the United States and Europe. However, these techniques are non-destructive, they can only be used for spot density (or air voids) measurements. Problems reported with these devices are usually caused by large variations in surface texture (Sebesta et al., 2013). In addition, there are particular concerns on using nuclear density gauge such as safety, costs associated with radioactive material licensing and requirements for closing off work areas.

This paper focuses on a case study of using Ground Penetrating Radar (GPR) as an alternate non-destructive evaluation of air voids content in newly constructed HMA. This technique has been used for the first time in the State of Qatar to determine the inplace air voids content of the HMA. The use of GPR as a rapid non-destructive technique can provide real-time information to paving contractors so that corrective action can be taken immediately. It must also be noted that GPR has been used for many years to determine asphalt layer thickness; however, this is the first time that this technique has been used as an alternative to coring and other non-destructive methods (e.g. nuclear and non-nuclear density gauges) to determine in-place air voids of HMA.

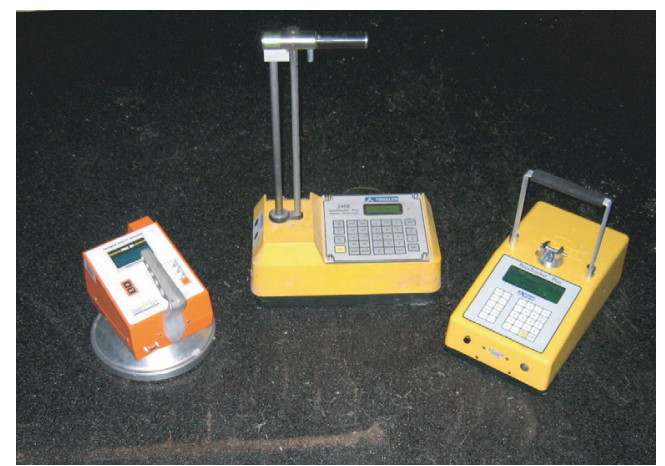

Figure 1: Nuclear density gauge and electromagnetic density indicators (Sebesta et al., 2013)

\section{METHODOLOGY}

GPR is an echo sounding method where a transducer (transmitter/receiver) is 
passed over the surface at a controlled speed. Short duration pulses of radio energy are transmitted into the subject and reflections from material boundaries and embedded features such as metalwork or voids are detected by the receiver. Sampling is so rapid that the collected data effectively constitutes a continuous cross section, enabling rapid assessment of density and condition over large areas. By assessing the amplitude, phase, and velocity of the signals from within the material, type and density of materials can be identified.

A case study was conducted on Al Khor Expressway utilizing a "GSSI PaveScan RDM" (Figure 2) GPR model integrated with GPS systems. The project deals with the construction of 34-km ten-lane dual carriageway expressway with 12 viaducts, eight road junctions, pedestrian footpaths, and two cycle lanes. Pavement construction consists of 3 asphalt layers (i.e. wearing course, base course class B, and base course class A) overlying roadbase and subbase layers, each approximately $150 \mathrm{~mm}$ thick and overlaying $500 \mathrm{~mm}$ of imported subgrade.

Pavescan RDM measurements are dielectric values. Dielectrics can be converted to physical properties (e.g. density and air voids content) by generating a least-squares fit relationship derived from dielectric values and known air voids content obtained over a range of dielectric values. Di-electric properties of HMA were determined through transmitted high frequency radio signal $(2 \mathrm{GHz}$ ) into the ground (few millimeters below the surface) and receiving pairs of the array. The data was collected at walking speed (approximately $1.5 \mathrm{~m} / \mathrm{s}$ ). The overspeed indicator in the status bar will turn red if the operator is walking too fast. A provisional standard has been developed by American Association of State and Highway Transportation Officials (AASHTO, 2019) on "Best Practice for Asphalt Surface Dielectric Profiling System using Ground Penetrating Radar" and the standard's procedure was followed in this study.

A series of core samples at known dielectrics were extracted from the site and air voids content of each core was determined in the laboratory. Air voids content (\%) vs. dielectric values were plotted and a least-squares-fit line was used to calculate the air voids content from subsequent dielectric values. The test was conducted on different layers (i.e. wearing course, base course class B, and base course class A as per (QCS, 2014)) and the measurement results on the wearing course are presented in the next section.

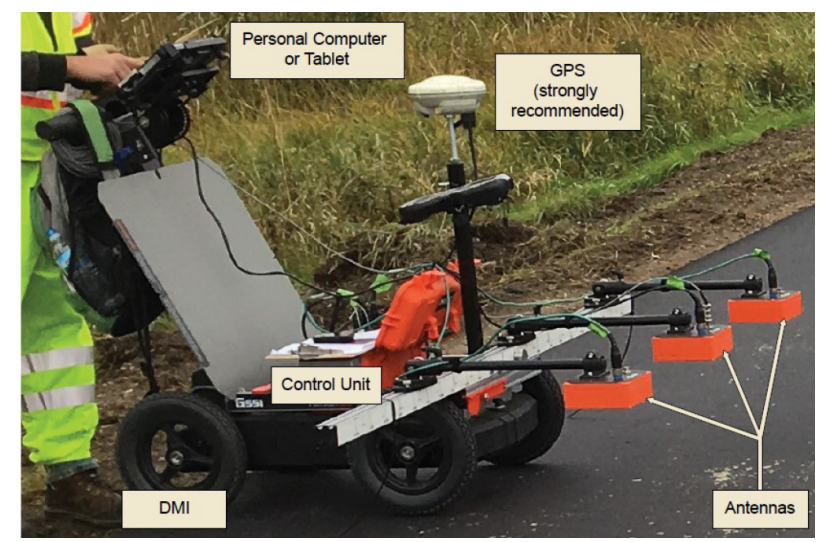

Figure 2: GSSI PaveScan RDM model with three antennas 0.5m apart 


\section{RESULTS AND DISCUSSION}

Figure 3 shows the relationship between dielectric readings taken from the GPR and air voids content measured on 39 extracted pair of core samples from the wearing course layer. A least-squares fit relationship with a coefficient of determination (i.e. R-square, $\mathrm{R}^{2}$ ) of 0.79 was generated.

The established relationship between di-electric and air voids was used to calculate the air voids content from subsequent dielectric values. Figure 4 shows an example of data collected on a section of the expressway using GSSI PaveScan RDM. Colorful graphs refer to data collected by 3 antennas. In order to validate the data, additional pairs of cores have been extracted from the site (under each antenna) and their air voids content measured in the laboratory. As shown in Figure 4, the measured air voids content of the core samples are in good agreement with collected GPR data. The difference in di-electric measurements between the 3 antennas, as it is clear in Lane 1 (Figure 4), can also be used as an indication of aggregate segregation on surface of the asphalt layer

Further analyses have been performed to establish the accuracy of the GPR data. A Root Mean Square Error (RMSE) of 0.55 was calculated which proves that the GPR tool can be used as a predictive tool to estimate the in-place air voids content of HMA with an average accuracy of \pm 0.55 . Further study needs to be performed to investigate the effect of HMA temperature, ambient temperature, and presence of moisture on the surface of HMA on the di-electric readings from the GPR.

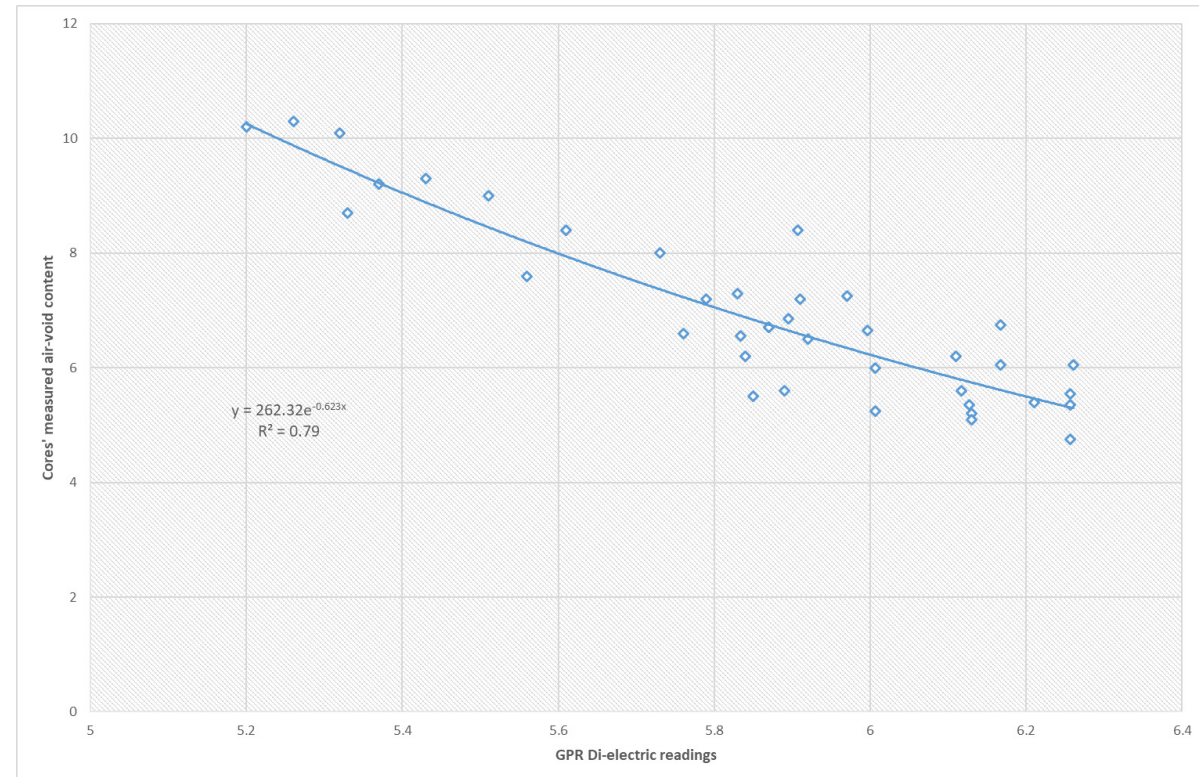

Figure 3: Relationship between GPR di-electric readings and air voids content of extracted cores 


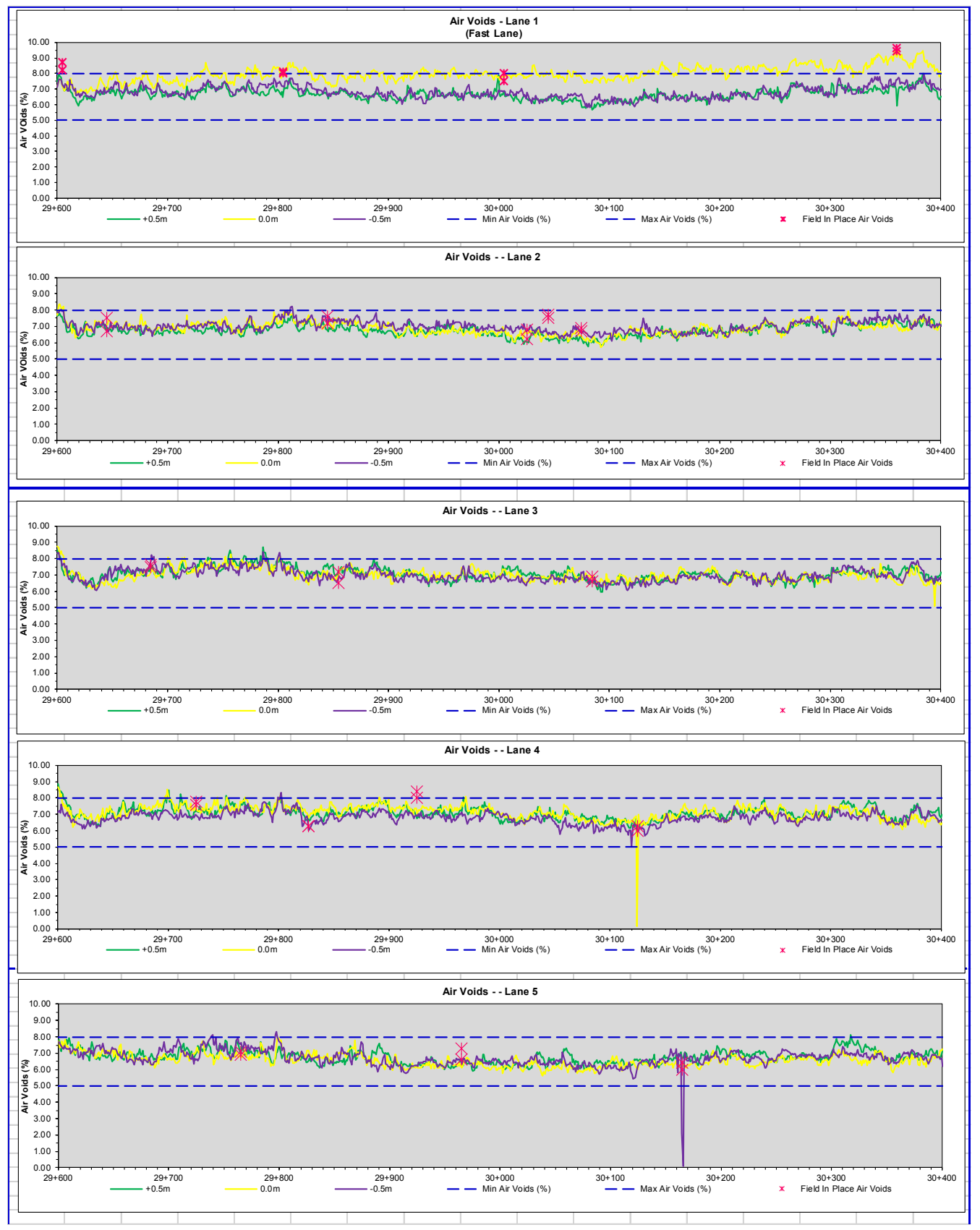

Figure 4: Example of data collected on a section of the expressway using GSSI PaveScan RDM

\section{CONCLUSION}

Obtaining adequate in-place air voids content is vital for achieving pavement durability for Hot-Mix Asphalt (HMA) pavement. A case study was carried out to use Ground Penetrating Radar (GPR) as an alternative non-destructive evaluation of air voids content in newly constructed HMA. "GSSI PaveScan RDM" GPR model integrated with 
GPS systems was utilized to determine in-place air voids content. The data collected by the GPR demonstrated good repeatability and correlation with cores' measured air voids content with a Root Mean Square Error (RMSE) of \pm 0.55 . This technique provides an opportunity for continuous measurement of air voids content in HMA and, at the same time, faster and reliable in-situ data for QA/QC which subsequently accelerate pavement construction significantly.

\section{REFERENCES}

American Association of State and Highway Transportation Officials (AASHTO) (2019). Asphalt Surface Dielectric Profiling System Using Ground Penetrating Radar. AASHTO PP 98-19, Washington, D.C., the USA.

Brown, E. R. (1990). Density of Asphalt Concrete - How Much Is Needed. Transportation Research Board 1282, TRB, National Research Council, Washington, D.C.

Linden, R.N., Mahoney, J. P. \& Jackson, N. C. (1989). Effect of Compaction on Asphalt Concrete Performance. Transportation Research Record 1217, TRB, National Research Council, Washington, D.C.

Qatar Construction Specifications (QCS) (2014). Section 6: Part 5, Roadworks, Ministry of Municipality and Environment.

Sebesta, S., Scullion, T. \& Saarenketo, T. (2013). Using Infrared and High-Speed GroundPenetrating Radar for Uniformity Measurements on New HMA Layers. (Report S2-R06CRR-1). Strategic Highway Research Program, National Transportation Board, Washington, D.C. 